\title{
RNA interference against viruses: strike and counterstrike
}

\author{
Joost Haasnoot, Ellen M Westerhout \& Ben Berkhout
}

RNA interference (RNAi) is a conserved sequence-specific, gene-silencing mechanism that is induced by double-stranded RNA. RNAi holds great promise as a novel nucleic acid-based therapeutic against a wide variety of diseases, including cancer, infectious diseases and genetic disorders. Antiviral RNAi strategies have received much attention and several compounds are currently being tested in clinical trials. Although induced RNAi is able to trigger profound and specific inhibition of virus replication, it is becoming clear that RNAi therapeutics are not as straightforward as we had initially hoped. Difficulties concerning toxicity and delivery to the right cells that earlier hampered the development of antisense-based therapeutics may also apply to RNAi. In addition, there are indications that viruses have evolved ways to escape from RNAi. Proper consideration of all of these issues will be necessary in the design of RNAi-based therapeutics for successful clinical intervention of human pathogenic viruses.

In recent years, known and emerging viruses have posed an increasingly serious threat to public health. Effective vaccines and antiviral drugs are not available for most of these viruses. RNAi has therefore been welcomed by the scientific community as a potentially powerful new tool to target viruses. Results from in vitro studies and animal models indicate that RNAi therapeutics can be highly effective at low dosage, which makes them outstanding candidates for future clinical use. Indeed, several RNAi-based antiviral drugs are currently being tested in clinical trials (Table 1). As such, the development of RNAi therapeutics is taking place at an unprecedented speed, moving from an obscure phenomenon reported in plants and Caenorhabditis elegans to therapeutic compounds in clinical trials in only a few years. Since the first report on RNAi-mediated inhibition of the human pathogen respiratory syncytial virus (RSV) in 2001 (ref. 1), many other viruses have been successfully targeted by RNAi (see review by J.H and B.B. ${ }^{2}$ ). These include, among others, important human pathogens, such as human immunodeficiency virus type $1(\mathrm{HIV}-1)^{3-7}$, hepatitis C virus $(\mathrm{HCV})^{8,9}$, hepatitis B virus $(\mathrm{HBV})^{10,11}$, severe acute respiratory syndrome coronavirus (SARS$\mathrm{CoV})^{12,13}$ and influenza $\mathrm{A}$ virus ${ }^{14}$.

RNAi technology is the latest in a long line of nucleic acid-based drug candidates that include antisense DNA, RNA decoys, ribozymes and aptamers (Fig. 1 and Table 1). The development of these other therapeutic modalities was severely hampered by practical problems, such as toxicity, instability in serum, and delivery. Over the years, some of these problems have been solved using chemically modified nucleic acids instead of unmodified DNA/RNA oligonucleotides ${ }^{15}$. Such modifications as phosphorothioate DNA, 2'-O-methyl RNA, peptide nucleic acids (PNAs), locked nucleic acids (LNAs) and morpholino phospho-

Laboratory of Experimental Virology, Department of Medical Microbiology, Center for Infection and Immunity Amsterdam (CINIMA), Academic Medical Center of the University of Amsterdam, Meibergdreef 15, 1105 AZ Amsterdam, The Netherlands. Correspondence should be addressed to B.B.

(b.berkhout@amc.uva.nl).

Published online 7 December 2007; doi:10.1038/nbt1369 roamidates increase the affinity of the oligonucleotides for their target sequence and increase serum stability ${ }^{16-19}$. Despite these intense efforts to test different chemical modifications, it has been extremely difficult to design potent antivirals that are not toxic to cells. An overview of antiviral nucleic acid therapies and clinical trials is provided in Table 1.

Although there are significant differences between the mechanisms and efficiency of antisense and RNAi-based therapeutic approaches, it is clear that the latter share many of the same problems with the former that will need to be resolved to achieve clinical success. Important concerns include viral escape from RNAi, off-target effects of RNAi treatment and the delivery of the RNAi-inducer to the right target cell.

This review discusses RNAi strategies to inhibit virus infections and describes viral countermeasures. Some viruses can escape from RNAi inhibition, which can be considered an active countermeasure or induced viral resistance. The replication cycle of a virus may also provide an intrinsic protection against RNAi attack, which we will classify as a passive countermeasure. Not all nucleic acid-based antiviral strategies are equally sensitive to these passive and active viral strategies, and we highlight the most promising therapeutic options.

\section{The RNAi mechanism}

To design potent RNAi-based antivirals, it is important to understand the underlying mechanisms and the function of RNAi in cell biology. RNAi plays a pivotal role in regulation of gene expression at the posttranscriptional level through microRNAs (miRNAs) ${ }^{20}$. miRNAs are small noncoding RNAs that are expressed as primary miRNAs and processed first by the protein Drosha and then by Dicer into a $\sim 70$ nucleotide (nt) stem-loop precursor miRNA (pre-miRNA) and the mature miRNA of 21-25 nt, respectively. One strand of the mature miRNA, the antisense or guide strand, is loaded into the RNA-induced silencing complex (RISC). The guide strand targets RISC to mRNAs with partially complementary sequences, triggering mRNA cleavage or translational inhibition. It has recently been suggested that under stress conditions, the mode of miRNA regulation can change and, by association with other pro- 


\section{Table 1 Nucleic acid-based antiviral therapeutics that have entered clinical trials}

\begin{tabular}{|c|c|c|c|c|c|}
\hline Virus & Inhibitor (name) & Target gene & Stage & Sponsor & Reference \\
\hline CMV & $\begin{array}{l}\text { Antisense oligonucleotide } \\
\text { (Vitravene; formivirsen/ISIS } \\
\text { 2922) }\end{array}$ & IE2 & Approved & Isis Pharmaceutials (Carlsbad, CA, USA) & $\begin{array}{l}126- \\
128\end{array}$ \\
\hline \multirow[t]{11}{*}{ HIV-1 } & \multirow[t]{2}{*}{ Ribozyme $^{b}(\mathrm{Rz} 2, \mathrm{OZ}-1)$} & \multirow[t]{2}{*}{ tat } & $\begin{array}{l}\text { Phase } 1 \text { complete, Phase } 2 \\
\text { ongoing }\end{array}$ & $\begin{array}{l}\text { Johnson \& Johnson (New Brunswick, NJ, } \\
\text { USA) subsidiary Tibotec Therapeutics } \\
\text { (Bridgewater, NJ, USA) }\end{array}$ & 129 \\
\hline & & & Phase 1 complete & Gene Shears and Johnson Research & 130 \\
\hline & $\begin{array}{l}\text { 937-nt antisense gene }{ }^{\mathrm{b}} \\
(\text { VRX496) }\end{array}$ & env & Phase 1 complete & VIRxSYS (Gaithersburg, MD, USA) & 131 \\
\hline & \multirow[t]{2}{*}{$\begin{array}{l}\text { Dominant-negative anti-HIV-1 } \\
\text { gene }^{\text {b }}(\text { RevM10) }\end{array}$} & \multirow[t]{2}{*}{ rev } & Phase $1 / 2$ ongoing & $\begin{array}{l}\text { Systemix (Palo Alto, CA, USA) and National } \\
\text { Cancer Institute (Bethesda, MD, USA) }\end{array}$ & 132,133 \\
\hline & & & Phase 1 complete & $\begin{array}{l}\text { The Saban Research Institute/USC Keck } \\
\text { School of Medicine (Los Angeles) }\end{array}$ & 134 \\
\hline & Decoy RNA ${ }^{b}$ & $R R E$ & Phase 1 complete & $\begin{array}{l}\text { Childrens Hospital Los Angeles, University } \\
\text { of Southern California School of Medicine } \\
\text { (Los Angeles, CA) and Baylor College of } \\
\text { Medicine (Houston) }\end{array}$ & 135 \\
\hline & $\begin{array}{l}\text { Short-hairpin RNA, ribozyme } \\
\text { and RNA decoyb (Triple-R } \\
\text { vector) }\end{array}$ & $\begin{array}{l}\text { tat/rev, CCR5, } \\
\text { TAR }\end{array}$ & Phase 1 complete & $\begin{array}{l}\text { Colorado State University (Fort Collins, } \\
\text { CO, USA) and Beckman Research Institute } \\
\text { (Duarte, CA, USA) }\end{array}$ & 136 \\
\hline & Antisense TAR and RevM10 ${ }^{\mathrm{b}}$ & $T A R$, rev & Phase 1 complete & $\begin{array}{l}\text { National Human Genome Research Institute } \\
\text { (Bethesda, MD, USA) }\end{array}$ & 137 \\
\hline & $\begin{array}{l}\text { Antisense oligonucleotide } \\
\text { (Gem 92) }\end{array}$ & Gag & Phase 2 discontinued & $\begin{array}{l}\text { Hybridon (now Idera Pharmaceuticals, } \\
\text { Cambridge, MA, USA) }\end{array}$ & NA \\
\hline & Antisense $^{\mathrm{b}}$ (HGTV43) & ND & Phase $1 / 2$ ongoing & Enzo Biochem (Farmingdale, NY, USA) & NA \\
\hline & Peptide nucleic acid (AVR-118) & ND & Phase $1 / 2$ completed & Advanced Viral Research (Yonkers, NY, USA) & NA \\
\hline RSV & $\begin{array}{l}\text { Small interfering RNA } \\
(A L N-R S V 01)\end{array}$ & Nucleocapsid & $\begin{array}{l}\text { Phase } 1 \text { ongoing and phase } \\
2 \text { planned for } 2008\end{array}$ & $\begin{array}{l}\text { Alnylam Pharmaceuticals (Cambridge, MA, } \\
\text { USA) }\end{array}$ & NA \\
\hline \multirow[t]{4}{*}{$\mathrm{HCV}$} & $\begin{array}{l}\text { Ribozyme } \\
\text { (Heptazyme) }\end{array}$ & IRES & $\begin{array}{l}\text { Phase } 2 \text { studies } \\
\text { discontinued }\end{array}$ & $\begin{array}{l}\text { Ribozyme Pharmaceuticals (Boulder, CO, } \\
\text { USA; renamed Sirna, now part of Merck) }\end{array}$ & 138 \\
\hline & $\begin{array}{l}\text { Antisense oligonucleotide }{ }^{a} \\
\text { (AVI-4065) }\end{array}$ & ND & $\begin{array}{l}\text { Phase } 2 \text { studies } \\
\text { discontinued }\end{array}$ & AVI BioPharma (Portland, OR, USA) & NA \\
\hline & \multirow[t]{2}{*}{$\begin{array}{l}\text { Antisense oligonucleotide }{ }^{a} \\
\text { (ISIS 14803) }\end{array}$} & \multirow[t]{2}{*}{ IRES } & $\begin{array}{l}\text { Phase } 2 \text { studies } \\
\text { discontinued }\end{array}$ & Isis & NA \\
\hline & & & Phase 1 completed & Isis & 139 \\
\hline HBV & $\begin{array}{l}\text { Short-hairpin RNA } \\
(\text { Nuc B1000) }\end{array}$ & $\begin{array}{l}\text { Pre-gen./pre-C, } \\
\text { Pre-S1, } \\
\text { Pre-S2/S, } X\end{array}$ & Phase 1 ongoing & Nucleonics (Horsham, PA, USA) & NA \\
\hline \multirow[t]{2}{*}{ HPV } & $\begin{array}{l}\text { Antisense oligonucleotide }{ }^{a} \\
\text { (MBI 1121) }\end{array}$ & E1 & Phase 1 discontinued & $\begin{array}{l}\text { Migenix (formerly Micrologix Biotech, } \\
\text { Vancouver, BC, Canada) }\end{array}$ & NA \\
\hline & Peptide nucleic acid (AVR-118) & ND & Phase 1 discontinued & Advanced Viral Research (Yonkers, NY, USA) & NA \\
\hline
\end{tabular}

${ }^{a}$ Chemically synthesized. ${ }^{b}$ Gene construct. ${ }^{~}$ Human papillomavirus. ND, not disclosed. NA, not available.

teins, can turn into an activator of gene expression ${ }^{21}$. It is currently estimated that expression of at least $30 \%$ of all human genes is regulated by miRNAs ${ }^{22}$. The exact criteria for target recognition are currently not clear. However, pairing of the $5^{\prime} 7-8$ nucleotides of the miRNA (seed region) to multiple sites in the $3^{\prime}$ untranslated region of a target mRNA is in many cases sufficient to trigger translational inhibition ${ }^{22-25}$.

RNAi is involved in the inhibition of viruses and silencing of transposable elements in plants, insects, fungi and nematodes by smallinterfering RNAs (siRNAs, 21-nt dsRNA) that are processed from dsRNA viral replication intermediates ${ }^{26-30}$. These siRNAs are loaded into RISC and target the fully complementary viral RNAs for destruction or translational repression ${ }^{31}$. Although still under debate, accumulating evidence suggests that RNAi also plays a role in the antiviral defense mechanisms in mammalian cells ${ }^{32-34}$. For example, the retrovirus primate foamy virus type 1 (PFV-1) and vesicular stomatitis virus (VSV) are inhibited by cellular miRNAs miR-32 and miR-24+ miR-93, respectively ${ }^{35,36}$. The expression of miR-24 and miR-93 is reduced in Dicer-deficient mice, causing a strong increase of VSV replication ${ }^{36}$. In addition, a recent paper indicates that cellular miRNAs miR-28, miR-125b, miR-150, miR-223 and miR-382 suppress the expression of HIV-1 mRNAs in resting CD4 ${ }^{+}$ $\mathrm{T}$ cells, suggesting that these miRNAs play a role in the establishment of viral latency ${ }^{37}$. Expression of these miRNAs is decreased in activated $\mathrm{CD} 4^{+} \mathrm{T}$ cells, allowing virus replication. These results are in agreement with the observation that the cellular miRNA expression pattern can be significantly influenced by virus infection ${ }^{38}$. In addition, the human retrotransposon LINE-1 (long interspersed nuclear element 1) is inhibited by transposon-specific siRNAs, similar to what has been described for transposon silencing in C. elegans ${ }^{39-41}$. Furthermore, data suggest the 


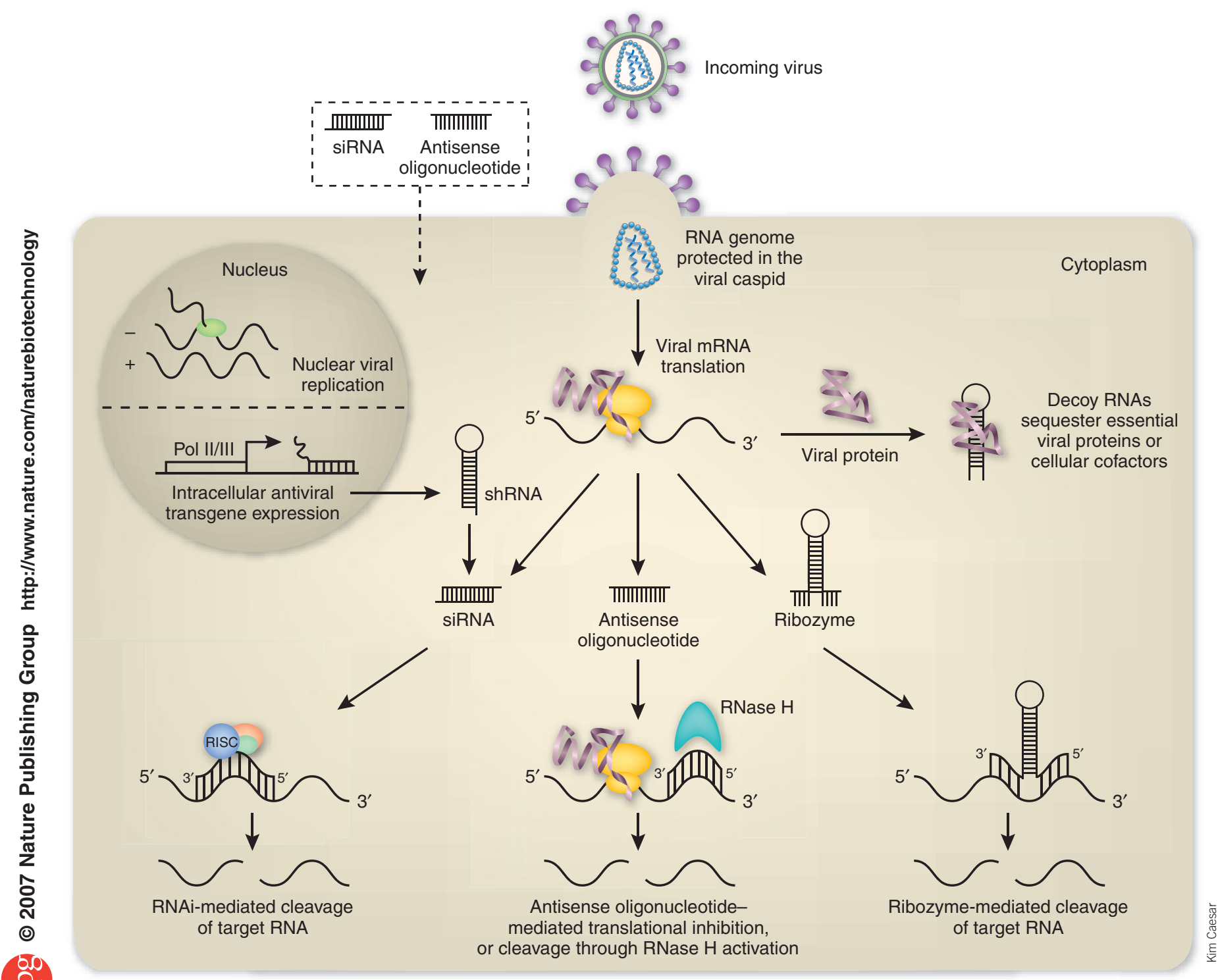

Figure 1 Nucleic acid-based antiviral strategies. Antiviral nucleic acids can either be transfected into cells (e.g., siRNA or antisense oligonucleotides) or expressed intracellularly (shRNA, ribozymes or RNA decoys). Viral transcripts complementary to the siRNA/shRNA are cleaved upon assembly of the RISC machinery. RISC is not able to target RNA genomes that are protected within viral capsids or shielded from RNAi attack in subcellular compartments (e.g., the nucleus or virus-induced vesicles). Modified antisense oligonucleotides have a high affinity for their target sequence and inhibit gene expression by steric hindrance of the ribosome, splicing (within the nucleus) or through induction of mRNA cleavage by recruitment of RNase $\mathrm{H}$. Binding of ribozymes to the target sequence should also trigger cleavage of the viral RNA. Decoy RNAs bind and sequester essential viral proteins or host cell factors that support virus replication.

accumulation of virus-specific siRNAs during HIV-1 replication ${ }^{32}$. We discuss below active and passive viral strategies that counter this antiviral RNAi response (summarized in Fig. 2).

Besides possible antiviral RNAi effects, it is clear that there is an intricate interplay between mammalian viruses and the host cell RNAi machinery ${ }^{42}$. Of particular note is the finding that the cellular miR-122 stimulates HCV replication through a yet-unknown mechanism ${ }^{43}$. In addition, various herpes viruses encode miRNAs that are thought to target specific cellular genes ${ }^{44-48}$. Herpes simplex virus type 1 (HSV-1) expresses the anti-apoptotic viral miR-LAT that inhibits the induction of apoptosis by downregulating the expression of transforming growth factor (TGF)- $\beta 1$ and SMAD3 (ref. 49). Simian virus 40 (SV40) encodes miRNAs that regulate viral gene expression, thus reducing recognition of infected cells by the immune system ${ }^{50}$.

\section{Virus-encoded suppressors of RNAi}

To counter the antiviral RNAi response, plant and invertebrate viruses have evolved proteins that actively interfere with distinct steps of the RNAi machinery to ensure high virus production and efficient viral spread $^{51}$. Suppressor activity has also been reported for mammalian viruses (Fig. 2). The primate retrovirus PFV-1 overcomes miRNAmediated antiviral pressure by RNAi suppressor activity of the Tas protein $^{35}$. Other mammalian viruses also encode viral factors that exhibit RNAi suppression activity: either such proteins as influenza A virus NS1, vaccinia virus E3L, HCV Core, PFV-1 Tas, HIV-1 Tat and the Ebola virus VP35 protein, or RNAs such as the adenovirus virus-associated RNAs I and II (VAI and VAII) ${ }^{32,35,52-56}$. Recently, we showed that VP35, E3L and NS1 can functionally replace the HIV-1 Tat protein to support virus production of a Tat-minus virus variant, suggesting that RNAi 
suppression is a common activity in mammalian viruses ${ }^{53}$. It is currently unclear to what extent these suppressors can interfere with the activity of RNAi-based therapeutics. Even so, the fact that viruses are efficiently inhibited by induced RNAi illustrates that viral-suppression activity does not pose a serious problem for therapeutic RNAi.

A single point mutation within the HIV-1 target sequence can trigger viral escape from RNAi-pressure. Apparently, inhibition by the translational component of the RNAi mechanism is not operational on such mismatched templates. RNAi suppression activity mediated by the HIV-1 Tat protein may help the virus to overcome translational repression by partially complementary siRNAs. This would be similar to what has been described for PFV-1, where the Tas protein suppresses the inhibitory effect induced by miR-32 (ref. 35).

\section{Strategies for inducing RNAi}

Elbashir and coworkers ${ }^{57}$ were the first to show that transfection of synthetic siRNAs could induce RNAi-mediated gene silencing in mammalian cells. These synthetic siRNA duplexes are generally 21-nt long with 2-nt 3 ' overhangs and are modeled after the natural Dicer cleavage products. Upon dsRNA transfection, the antisense strand of the siRNA is loaded into RISC, which can subsequently target the viral RNA in a sequence-specific manner. Similar to antisense oligonucleotides, synthetic siRNAs are relatively unstable in vivo due to degradation by nucleases. Chemical modifications that were previously used to increase the in vivo half-life of antisense compounds have been used to stabilize siRNAs ${ }^{58-60}$. However, these modifications can have a negative effect on siRNA activity ${ }^{61}$. In addition, enhanced stability or activity in vitro may not translate into more active compounds in vivo ${ }^{62}$.

Transfection of plasmids that express antiviral short-hairpin RNAs (shRNAs) is also commonly used to induce RNAi in mammalian cells ${ }^{63}$. These 19-29 base pair (bp) shRNAs are modeled after pre-miRNAs with a small apical loop and a 3'-terminal UU overhang. Short-hairpin RNAs are expressed in the nucleus from a polymerase III promoter, translocated to the cytoplasm by Exportin-5, and further processed by Dicer in the cytoplasm into functional siRNAs. Recently, the activity of shRNAs has been significantly improved by inclusion of RNA structure motifs that mimic natural miRNAs. These improved shRNAs (shRNA-mirs) are expressed as larger transcripts and contain bulged nucleotides and large loops, mimicking the structure of primary or premiRNAs ${ }^{64-67}$.

Another way to induce RNAi is by intracellular expression of longhairpin RNAs (lhRNAs) ${ }^{68-73}$. In contrast to transfection of dsRNA larger than $30 \mathrm{bp}$, intracellular expression does not seem to induce the interferon response. Efficient inhibition by lhRNAs has been reported for HIV-1, HCV and HBV. The potential advantage of a lhRNA inhibitor is the generation of multiple siRNAs from a single precursor molecule, which may prevent viral escape.

\section{Targets for antiviral RNAi strategies}

For potent and durable inhibition of virus replication, it is important to target the viral RNA genome or those transcripts that encode essential viral factors. In addition, it is important to target sequences that are conserved among different virus strains to reduce the chance of escape. Nevertheless, viruses tend to escape from RNAi inhibition by mutating the target sequence $\mathrm{e}^{74-79}$. Host factors that are essential for viral replication can also be targeted, and this approach may reduce the chance of viral escape. However, there are few host factors that can be targeted without affecting host cell viability ${ }^{7,80-83}$. A well-known example of one such host factor is the CCR5 (C-C motif receptor 5) coreceptor for HIV-1, which is important for virus entry, yet mutation of the CCR5 gene is known to be compatible with normal life 84,85 .
As we learn more about viruses and their interactions with the cellular RNAi pathway, new targets for antiviral therapeutics may become evident. As discussed above, HCV requires miR-122 for its replication ${ }^{43}$. Possibly, this miR-122 cofactor could be targeted with antagomirs, antisense oligonucleotides that specifically inhibit miRNA function. Because miRNA function is believed to be highly redundant, silencing of a single miRNA could be well tolerated by a cell. HIV-1 inhibits the expression of miR-20 and miR-17-5p, resulting in increased histone acetylase PCAF (P300/CRE (cAMP response element) binding protein (CBP)-associated factor) expression, which is required for optimal HIV-1 transcription ${ }^{86}$. Overexpression of miR20 has been shown to reduce PCAF expression, resulting in reduced virus replication. But overexpression of natural miRNAs may possibly result in less unwanted side effects than is the case with man-made siRNAs. If cellular miRNAs can indeed target viral mRNAs, silencing of the viral RNAi suppressor could de-repress these miRNAs, resulting in a multiple miRNA attack ${ }^{35,36}$. Although there is no consensus, several reports suggest that, similar to the situation in plants, exogenous siRNAs can trigger promoter methylation in a sequencespecific manner in mammalian cells ${ }^{87-90}$. The use of siRNAs to trigger transcriptional silencing may represent an alternative RNAi inhibition strategy to target DNA and retroviruses.

\section{Transient RNAi strategies to target acute virus infections}

Transient transfection of synthetic siRNAs or plasmids encoding shRNAs is probably best suited for the treatment of acute virus infections. Respiratory viruses are ideal targets for this therapy approach because the upper airways and lungs are relatively easy to target. The RNAi therapeutic compounds could be administered via aerosol delivery devices similar to the ones used for delivery of asthma therapeutics. Even a partial reduction of the peak viral load will significantly reduce or avoid disease symptoms, and the virus is cleared thereafter by the immune system. Mice treated with siRNAs against influenza virus, RSV and SARS-CoV showed reduced virus titers and reduced virus-induced mortality, both as a prophylactic and in treatment of established infections ${ }^{13,91,92}$. Interestingly, it has been reported that siRNA treatment is effective both with and without transfection reagents ${ }^{92}$. Intranasal administration of plasmids expressing shRNA against RSV also resulted in a significant decrease of viral titers ${ }^{93}$. Potent siRNA inhibitors against the SARS-CoV spike and polymerase genes have been shown to reduce SARS-like symptoms, viral RNA levels and lung histopathology in rhesus macaque ${ }^{13}$. Recently, Alnylam Pharmaceuticals (Cambridge, MA, USA) announced that it has initiated a phase 2 clinical trial with an siRNA for the treatment of RSV (Table 1). In the phase 1 trial, no adverse effects were observed, and now the safety, tolerability and antiviral activity of the siRNA will be tested in adults experimentally infected with RSV.

\section{Persistent viral infections: RNAi therapy and virus escape}

In contrast to acute virus infections, chronic infections with such viruses as HIV-1, HCV and HSV should be targeted with a long-term RNAi treatment. In this scenario, gene therapy approaches are needed to provide a constant supply of intracellularly expressed antiviral shRNAs. In the case of $\mathrm{HIV}-1$, one could make virus-resistant $\mathrm{CD} 4^{+} \mathrm{T}$ cells by ex vivo transduction of blood stem cells to express the anti-HIV-1 RNAi trigger and give these cells back to the patient ${ }^{94}$. Different viral vectors have been used to stably transduce cells with shRNA expression constructs. For HIV-1, we have shown that virus replication is strongly inhibited in cells transduced with a lentiviral shRNA vector ${ }^{79,95,96}$. Unfortunately, prolonged culturing of these cells results in the selection of escape variants that become resistant to the expressed siRNA. The induced RNAi block of HIV-1 replication is likely not to be absolute, allowing escape variants to evolve. These resistant variants contain a single nucleotide 


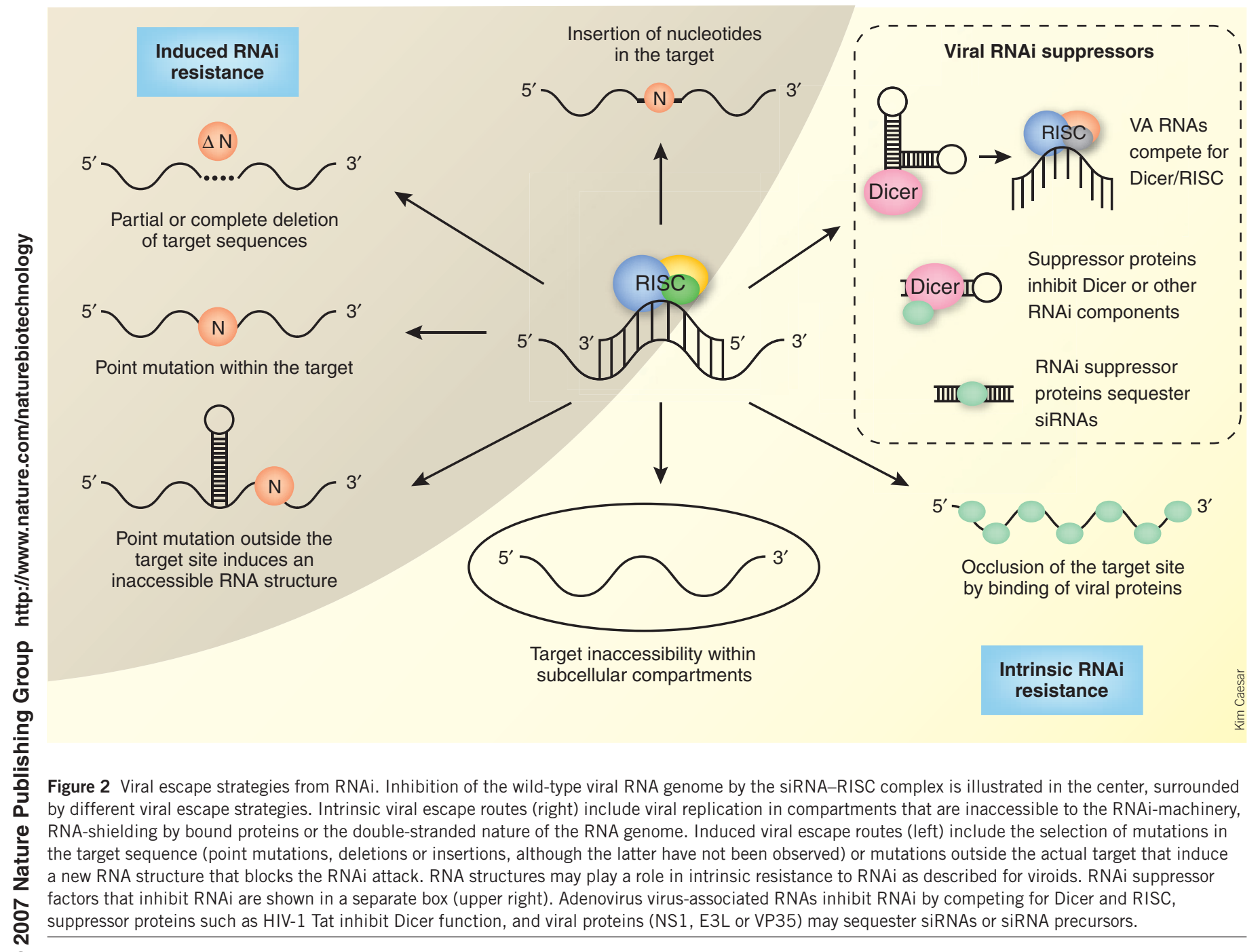

substitution or deletion within the siRNA-target sequence (Fig. 2) $)^{74,79}$. The acquisition of deletions was reported when the nonessential Nef gene was targeted, but this escape route does not occur when essential viral genes are targeted (K. van Eije, O. ter Brake and B.B., unpublished data). B.B and colleagues ${ }^{97}$ have suggested that theoretically, viruses can also escape from drug-pressure by selection of sequence insertions, but this has not yet been reported for the inhibition of RNAi. Similar escape by target-site mutations has been reported for other viruses, such as poliovirus ${ }^{77}$. This suggests that a single nucleotide substitution in the targeted sequence is sufficient to overcome the antiviral activity of siRNAs, although more mutations may be required for full resistance. These results confirm the exquisite sequence-specificity of RNAi.

Besides escape variants with mutations in the 19-nt target, our group has identified an HIV-1 variant that obtained resistance by selection of an upstream mutation ${ }^{95}$. This mutation induces an alternative local RNA structure in which the target sequence, in particular its $3^{\prime}$ end, is occluded from siRNA/RISC binding. This result highlights the impact of target RNA structure on RNAi and indicates an alternative way for viruses to evade RNAi-mediated inhibition. To design potent antiviral RNAi therapeutics, one must investigate the local RNA secondary structure of the target sequence. The protective role of RNA structure is discussed in more detail below when we describe intrinsic viral replication properties that avoid RNAi attack (Fig. 2).
Thus, a single siRNA therapy is not sufficient to obtain long-term inhibition of virus replication. Escape from RNAi is reminiscent of the evolution of drug-resistant HIV-1 variants in individuals on antiretroviral therapy. Only the combined use of multiple antiviral drugs can permanently block virus replication and prevent the emergence of resistant variants. Similarly, the combined expression of multiple siRNAs would be required to persistently block virus replication ${ }^{96,98}$. This combinatorial approach should target viral sequences that are essential and well conserved among different virus strains. Even so, overexpression of multiple shRNAs increases the chance for off-target effects and toxicity by saturating the cellular RNAi pathway ${ }^{99,100}$. Saturating concentrations of siRNA/shRNAs may interfere with cellular miRNA processing and function; this has been shown to have potentially fatal consequences for mice treated with a viral vector overexpressing shRNAs in the liver ${ }^{101}$.

\section{RNAi insensitivity due to viral RNA structure}

Viruses can escape induced RNAi-mediated inhibition by evolving an alternative RNA structure that shields the target sequence ${ }^{95}$. This finding suggests that viruses may use highly structured RNAs as a means to evade the natural antiviral RNAi response. This has indeed been described for plant viroids ${ }^{102,103}$. These pathogens have a small (200-400 nt), noncoding, single-stranded circular RNA genome that adopts a quasi rodshaped structure by intramolecular base pairing ${ }^{104}$. Because viroids do 
not encode proteins, they cannot evolve protein suppressors to counteract RNAi induced by the dsRNA nature of the viroid genome (Fig. 2). Therefore, viroids may have evolved their structure-based replication strategy to become inaccessible to RISC and resistant to RNAi ${ }^{102}$. The circular genomic and antigenomic RNA of hepatitis delta virus (HDV), which requires HBV as a helper virus for its replication, are RNAi resis$\operatorname{tant} t^{105}$. This resistance was ascribed to inaccessibility based on their nuclear localization. But even when the genomic RNA enters the cytoplasm, it will be inaccessible to RNAi attack because of its viroid-like structure (74\% base pairing) or alternatively through binding of a host RNA-binding protein ${ }^{105}$.

Another example of RNAi resistance based on RNA structure was described for the $3^{\prime}$ - and $5^{\prime}$-untranslated regions of the rhinovirus RNA genome. RNAi targeted to these regions was ineffective, probably because of the stable RNA structure of these genome ends. Similar results were obtained when Gitlin et al. ${ }^{77}$ targeted the well conserved and highly structured 5 ' noncoding region of the poliovirus RNA genome ${ }^{77}$. As we have noted, RNA-protein interactions may also negatively influence RNAi sensitivity ${ }^{106-108}$.

\section{Intrinsic virus replication strategies to avoid RNAi attack}

For optimal inhibition of virus replication it is important to target the virus at the early stages of virus replication. Inevitably, RNAi can only target viral RNAs after the virus has entered the cell. In the case of RNA viruses, the 'incoming' RNA genome would be an important target (Fig. 1). However, it turns out that viral genomic RNAs are difficult to target with RNAi. The RNA genome of retroviral particles seems an ideal target for RNAi before it is reverse transcribed into DNA, but the nucleocapsid particle of Rous sarcoma virus shields the incoming viral RNA from RNAi-mediated degradation ${ }^{109}$. There have been contradictory reports of whether the incoming HIV-1 RNA genome can be targeted by RNAi ${ }^{3,4,109-113}$. We addressed this issue using HIVbased lentiviral transduction as a quantitative model for HIV-1 infection and found no targeting ${ }^{114}$. We argued that the cytoplasmic core particle is likely to be inaccessible to RISC, but complete coverage of the viral RNA genome by nucleocapsid protein may also help to resist RNAi attack. The ability of HIV-1 to evade RNAi-mediated targeting of the incoming genome will frustrate therapeutic actions to prevent the establishment of an integrated DNA provirus. In other words, all therapeutic effects will be due to targeting of newly synthesized viral transcripts and inhibition of virus production. It will therefore be difficult to tailor RNAi strategies in a prophylactic HIV-1 vaccine that prevents infection.

In the initial experiments with RNAi-mediated inhibition of RSV, it was observed that the negative-strand genomic RNA is not targeted ${ }^{1}$. RSV protects its cytoplasmic genome by nucleoprotein oligomerization along the viral RNA, forming a ring structure in which the RNA is sequestered and thereby protected against RISC with antiviral siRNAs. Similar structures have recently been reported for rabies virus and VSV ${ }^{115,116}$. Even when the viral RNA genome is covered by viral chaperonin proteins, which are known to facilitate RNA-RNA interactions, there are no indications that this class of RNA-binding proteins can stimulate the annealing of siRNA-RISC complexes.

RNA replication intermediates that are located in the cytoplasm are theoretical targets for RISC, whereas nuclear RNA molecules may be protected from the RNAi machinery (Fig. 2). For example, the influenza virus genomic RNAs localize in the nucleus and are therefore resistant to RNAi, whereas the cytoplasmic mRNA molecules are efficiently targeted $^{91}$. Reoviruses are dsRNA viruses that replicate in viral inclusions that form in the cytoplasm of infected cells ${ }^{117}$. The dsRNA genome that resides in these inclusions is protected against RNAi ${ }^{118}$. Flaviviruses reorganize the endoplasmic reticulum membrane, such that its RNA genome is protected against RNAi attack ${ }^{119}$. Silencing of picornavirus is efficient when targeting the positive-strand RNAs, not the negativestrand RNAs ${ }^{120}$. This might indicate that the negative-strand RNAs are protected within the membrane-associated replication complexes ${ }^{121}$, whereas the positive-strand RNAs leave this complex to function as mRNA, which makes them susceptible to the RISC-machinery.

\section{Viral escape options for non-RNAi antiviral nucleic acids}

In contrast to RNAi strategies, little is known about viral escape options for other nucleic acid-based therapeutics. Inhibition of gene expression by antisense approaches does not rely on recruitment of a cellular silencing mechanism. Instead, the inhibitory effect is largely based on strong binding of antisense oligonucleotides to the target mRNA, which can block splicing events or the elongating ribosome through steric hindrance ${ }^{122}$. In addition, unmodified DNA or phosphorothioate-modified oligonucleotides may induce RNA cleavage by activation of cellular RNase $\mathrm{H}$, the enzyme that specifically recognizes DNARNA duplexes. Escape from antisense inhibition has been reported for SARS-CoV inhibited with morpholino-modified oligonucleotides ${ }^{123}$. The antisense oligonucleotide targeted the transcription-regulatory sequence, which is important for the process of discontinuous transcription. Mutation of a CUC motif into AAA enabled the virus to restore virus replication. However, virus replication was still strongly attenuated, which might indicate that escape from antisense therapeutics is not as easy as escape from RNAi. There are no data available on virus escape from antiviral ribozymes; however, point mutations in the target sequences will prevent efficient binding of the ribozyme and therefore result in escape. Decoy and aptamers that bind and sequester viral factors are not very likely to suffer from escape, although detailed studies are lacking. The absence of experimental evidence may be taken as a positive sign for these antiviral strategies, but care should be taken, as weak or nonspecific inhibitors will not trigger the selection of escape viruses.

\section{Strategies to counter viral RNAi evasion mechanisms}

Not all viral RNAs are equally sensitive to RNAi attack. This may be the result of protective proteins that bind to the viral RNA, protective RNA structure or because the viral RNA resides in a virus particle or a subcellular compartment that is not surveyed by the RNAi machinery (Fig. 2). It seems difficult, if not impossible, to redirect RNAi to these compartments. An interesting approach to target the retroviral RNA genome within virus particles is by means of specific antisense oligonucleotides that instruct the intravirion RNase H enzyme to cleave the viral genome ${ }^{124}$. Special countermeasures can also be designed to neutralize other viral evasion mechanisms. For example, the use of multiple effective shRNAs (or a single lhRNA that produces multiple antiviral siRNAs) in a combination therapy will not only give additive inhibition, but will also raise the genetic threshold for escape because multiple targets should acquire mutations before viral escape is apparent ${ }^{96}$. As an additional benefit, such escape variants may have reduced viral fitness, in particular when these variants acquire mutations in sequences that are highly conserved among virus isolates.

When preferred viral escape routes are frequently observed with a single shRNA inhibitor, one could consider a multiple shRNA approach in which these escape mutations are anticipated and selectively countered by other shRNAs, as we have previously outlined ${ }^{125}$. The inclusion of cellular cofactors as RNAi target is also likely to make viral escape more difficult. Possibly, viral sequences that are protected by stable RNA structure may be targeted by modified siRNAs with duplex-invading properties that bind with high affinity to the target. 
Viral proteins may frustrate an RNAi attack by acting as RNAi suppressor or by binding the RNA target. In such cases, one may consider targeting the viral mRNAs that encode these proteins.

\section{Conclusions}

Recent studies have underscored the importance of RNAi in eukaryotic cell biology. Antiviral RNAi strategies co-opt existing mechanisms and may therefore be more efficient than other nucleic acid-based antivirals. Multiple clinical RNAi applications are currently being tested, from a transient therapy for RSV to a durable gene therapy for HIV-1. However, we should take into account that viruses and host cells have co-evolved for millions of years and viruses have developed mechanisms to escape from RNAi. Thus, a better understanding of natural virus-RNAi interactions is key for the development of an effective RNAi-based antiviral drug. Improvement of the activity, specificity and delivery of RNAi inducers is a critical first step, followed by a detailed screen for unwanted side effects. It is possible that more potent antivirals can be constructed by designing miRNA-like transcripts. Not all viral targets are equally suitable or effectively inhibited by RNAi, and it will be important to identify the viral Achilles heel for an RNAi attack. Given the immense interest in RNAi as a therapeutic modality, the coming years are likely to see an increasing range of clinical applications. The realization of the potential of RNAi therapies to address human viral pathogens suggests that this field has a very promising future.

\section{ACKNOWLEDGMENTS}

We thank Walter de Vries for critically reading the manuscript and stimulating discussions. RNAi research in the Berkhout laboratory is sponsored by ZonMw (Vici grant and Translational Gene Therapy program), NWO-CW (Top grant), the European Union (LSHP-CT-2006-037301) and the Technology Foundation STW (grant AGT.7708).

Published online at http://www.nature.com/naturebiotechnology/

Reprints and permissions information is available online at http://npg.nature.com/ reprintsandpermissions/

1. Bitko, V. \& Barik, S. Phenotypic silencing of cytoplasmic genes using sequencespecific double-stranded short interfering RNA and its application in the reverse genetics of wild type negative-strand RNA viruses. BMC Microbiol. 1, 34 (2001).

2. Haasnoot, P.C.J. \& Berkhout, B. RNA interference: its use as antiviral therapy in Handbook of Experimental Pharmacology Vol. 173, 117-150 (Springer, Berlin and Heidelberg, 2006).

Coburn, G.A. \& Cullen, B.R. Potent and specific inhibition of human immunodeficiency virus type 1 replication by RNA interference. J. Virol. 76, 9225-9231 (2002).

4. Jacque, J.M., Triques, K. \& Stevenson, M. Modulation of HIV-1 replication by RNA interference. Nature 418, 435-438 (2002).

5. Lee, N.S. et al. Expression of small interfering RNAs targeted against HIV-1 rev transcripts in human cells. Nat. Biotechnol. 20, 500-505 (2002).

6. Novina, C.D. et al. siRNA-directed inhibition of HIV-1 infection. Nat. Med. 8, 681-686 (2002).

7. Qin, X.F., An, D.S., Chen, I.S.Y. \& Baltimore, D. Inhibiting HIV-1 infection in human T cells by lentiviral-mediated delivery of small interfering RNA against CCR5. Proc. Natl. Acad. Sci. USA 100, 183-188 (2003).

8. Kronke, J. et al. Alternative approaches for efficient inhibition of hepatitis $C$ virus RNA replication by small interfering RNAs. J. Virol. 78, 3436-3446 (2004).

9. Yokota, T. et al. Inhibition of intracellular hepatitis $C$ virus replication by synthetic and vector-derived small interfering RNAs. EMBO Rep. 4, 602-608 (2003).

10. McCaffrey, A.P. et al. Inhibition of hepatitis B virus in mice by RNA interference. Nat. Biotechnol. 21, 639-644 (2003).

11. Shlomai, A. \& Shaul, Y. Inhibition of hepatitis B virus expression and replication by RNA interference. Hepatology 37, 764-770 (2003).

12. He, M.L. et al. Inhibition of SARS-associated coronavirus infection and replication by RNA interference. J. Am. Med. Assoc. 290, 2665-2666 (2003).

13. Li, B.J. et al. Using siRNA in prophylactic and therapeutic regimens against SARS coronavirus in Rhesus macaque. Nat. Med. 11, 944-951 (2005).

14. Ge, $Q$. et al. RNA interference of influenza virus production by directly targeting mRNA for degradation and indirectly inhibiting all viral RNA transcription. Proc. Natl. Acad. Sci. USA 100, 2718-2723 (2003).

15. Kurreck, J. Antisense technologies. Improvement through novel chemical modifications. Eur. J. Biochem. 270, 1628-1644 (2003).

16. Larsen, H.J., Bentin, T. \& Nielsen, P.E. Antisense properties of peptide nucleic acid. Biochim. Biophys. Acta 1489, 159-166 (1999).
17. Petersen, M. \& Wengel, J. LNA: a versatile tool for therapeutics and genomics. Trends Biotechnol. 21, 74-81 (2003).

18. Iversen, P.L. Phosphorodiamidate morpholino oligomers: favorable properties for sequence-specific gene inactivation. Curr. Opin. Mol. Ther. 3, 235-238 (2001).

19. Jakobsen, M.R., Haasnoot, J., Wengel, J., Berkhout, B. \& Kjems, J. Efficient inhibition of HIV-1 expression by LNA modified antisense oligonucleotides and DNAzymes targeted to functionally selected binding sites. Retrovirology 4, 29 (2007).

20. Bartel, D.P. MicroRNAs: genomics, biogenesis, mechanism, and function. Cel/ 116 , 281-297 (2004).

21. Leung, A.K. \& Sharp, P.A. microRNAs: a safeguard against turmoil? Cell 130 581-585 (2007).

22. Krek, A. et al. Combinatorial microRNA target predictions. Nat. Genet. 37, 495-500 (2005).

23. Brennecke, J., Stark, A., Russell, R.B. \& Cohen, S.M. Principles of microRNA-target recognition. PLoS Biol. 3, e85 (2005).

24. Grimson, A. et al. MicroRNA targeting specificity in mammals: determinants beyond seed pairing. Mol. Cell 27, 91-105 (2007)

25. Lewis, B.P. Shih, I.H Jones-Rhoades, M.W., Bartel, D.P \& Burge, C.B. Prediction of mammalian microRNA targets. Cell 115, 787-798 (2003).

26. Waterhouse, P.M., Wang, M.B. \& Lough, T. Gene silencing as an adaptive defence against viruses. Nature 411, 834-842 (2001).

27. Voinnet, O. RNA silencing as a plant immune system against viruses. Trends Genet 17, 449-459 (2001)

28. Wilkins, C. et al. RNA interference is an antiviral defence mechanism in Caenorhabditis elegans. Nature 436, 1044-1047 (2005).

29. Wang, X.H. et al. RNA interference directs innate immunity against viruses in adult Drosophila. Science 312, 452-454 (2006).

30. Segers, G.C., Zhang, X., Deng, F., Sun, Q. \& Nuss, D.L. Evidence that RNA silencing functions as an antiviral defense mechanism in fungi. Proc. Natl. Acad. Sci. USA 104, 12902-12906 (2007).

31. Haasnoot, P.C.J., Cupac, D. \& Berkhout, B. Inhibition of virus replication by RNA interference. J. Biomed. Sci. 10, 607-616 (2003)

32. Bennasser, Y., Le, S.Y., Benkirane, M. \& Jeang, K.T. Evidence that HIV-1 encodes an siRNA and a suppressor of RNA silencing. Immunity 22, 607-619 (2005).

33. Cullen, B.R. Is RNA interference involved in intrinsic antiviral immunity in mammals? Nat. Immunol. 7, 563-567 (2006).

34. Berkhout, B. \& Jeang, K.T. RISCy business: microRNAs, pathogenesis, and viruses J. Biol. Chem. 282, 26641-26645 (2007).

35. Lecellier, C.H. et al. A cellular microRNA mediates antiviral defense in human cells Science 308, 557-560 (2005).

36. Otsuka, M. et al. Hypersusceptibility to vesicular stomatitis virus infection in Dicer 1 deficient mice is due to impaired miR24 and miR93 expression. Immunity 27 , 123-134 (2007).

37. Huang, J. et al. Cellular microRNAs contribute to HIV-1 latency in resting primary CD4(+) T lymphocytes. Nat. Med. 13, 1241-1247 (2007)

38. Yeung, M.L. et al. Changes in microRNA expression profiles in HIV-1-transfected human cells. Retrovirology 2, 81 (2005).

39. Sijen, T. \& Plasterk, R.H. Transposon silencing in the Caenorhabditis elegans germ line by natural RNAi. Nature 426, 310-314 (2003).

40. Soifer, H.S., Zaragoza, A., Peyvan, M., Behlke, M.A. \& Rossi, J.J. A potential role for RNA interference in controlling the activity of the human LINE-1 retrotransposon. Nucleic Acids Res. 33, 846-856 (2005)

41. Yang, N. \& Kazazian, H.H. Jr. L1 retrotransposition is suppressed by endogenously encoded small interfering RNAs in human cultured cells. Nat. Struct. Mol. Biol. 13, 763-771 (2006).

42. Cullen, B.R. Viruses and microRNAs. Nat. Genet. 38 (Suppl.), S25-S30 (2006).

43. Jopling, C.L., Yi, M., Lancaster, A.M., Lemon, S.M. \& Sarnow, P. Modulation of hepatitis C virus RNA abundance by a liver-specific microRNA. Science 309, 1577-1581 (2005).

44. Cai, X. et al. Epstein-Barr virus microRNAs are evolutionarily conserved and differentially expressed. PLoS Pathog. 2, e23 (2006).

45. Cui, C. et al. Prediction and identification of herpes simplex virus 1-encoded micro RNAs. J. Virol. 80, 5499-5508 (2006).

46. Grundhoff, A., Sullivan, C.S. \& Ganem, D. A combined computational and microarray-based approach identifies novel microRNAs encoded by human gammaherpesviruses. RNA 12, 733-750 (2006).

47. Pfeffer, S. et al. Identification of microRNAs of the herpesvirus family. Nat. Methods 2, 269-276 (2005).

48. Pfeffer, S. et al. Identification of virus-encoded microRNAs. Science 304, 734-736 (2004).

49. Gupta, A., Gartner, J.J., Sethupathy, P., Hatzigeorgiou, A.G. \& Fraser, N.W. Antiapoptotic function of a microRNA encoded by the HSV-1 latency-associated tran script. Nature 442, 82-85 (2006).

50. Sullivan, C.S., Grundhoff, A.T., Tevethia, S., Pipas, J.M. \& Ganem, D. SV40-encoded microRNAs regulate viral gene expression and reduce susceptibility to cytotoxic T cells. Nature 435, 682-686 (2005)

51. Li, F. \& Ding, S.W. Virus counterdefense: diverse strategies for evading the RNA silencing immunity. Annu. Rev. Microbiol. 60, 503-531 (2006).

52. Andersson, M.G. et al. Suppression of RNA interference by adenovirus virusassociated RNA. J. Virol. 79, 9556-9565 (2005).

53. Haasnoot, J. et al. The Ebola virus VP35 protein is a suppressor of RNA silencing. PLoS Pathog. 3, e86 (2007).

54. Li, W.X. et al. Interferon antagonist proteins of influenza and vaccinia viruses are sup pressors of RNA silencing. Proc. Natl. Acad. Sci. USA 101, 1350-1355 (2004). 
55. Lu, S. \& Cullen, B.R. Adenovirus VA1 noncoding RNA can inhibit small interfering RNA and microRNA biogenesis. J. Virol. 78, 12868-12876 (2004).

56. Wang, $Y$. et al. Hepatitis $C$ virus core protein is a potent inhibitor of RNA silencingbased antiviral response. Gastroenterology 130, 883-892 (2006).

57. Elbashir, S.M. et al. Duplexes of 21-nucleotide RNAs mediate RNA interference in cultured mammalian cells. Nature 411, 494-498 (2001).

58. Morrissey, D.V. et al. Potent and persistent in vivo anti-HBV activity of chemically modified siRNAs. Nat. Biotechnol. 23, 1002-1007 (2005).

59. Morrissey, D.V. et al. Activity of stabilized short interfering RNA in a mouse model of hepatitis B virus replication. Hepatology 41, 1349-1356 (2005).

60. Rana, T.M. Illuminating the silence: understanding the structure and function of small RNAs. Nat. Rev. Mol. Cell Biol. 8, 23-36 (2007).

61. Al Structural variations and stabilising modifications of synthetic siRNAs in mammalian cells. Nucleic Acids Res. 31, 2705-2716 (2003).

62. Layzer, J.M. et al. In vivo activity of nuclease-resistant siRNAs. RNA 10, 766-771 (2004)

63. Brummelkamp, T.R., Bernards, R. \& Agami, R. A system for stable expression of short interfering RNAs in mammalian cells. Science 296, 550-553 (2002).

64. Boden, D. et al. Enhanced gene silencing of HIV-1 specific siRNA using microRNA designed hairpins. Nucleic Acids Res. 32, 1154-1158 (2004).

65. Du, G., Yonekubo, J., Zeng, Y., Osisami, M. \& Frohman, M.A. Design of expression vectors for RNA interference based on miRNAs and RNA splicing. FEBS J. 273, 5421-5427 (2006).

66. Silva, J.M. et al. Second-generation shRNA libraries covering the mouse and human genomes. Nat. Genet. 37, 1281-1288 (2005).

67. Lo, H.L. et al. Inhibition of HIV-1 replication with designed miRNAs expressed from RNA polymerase II promoters. Gene Ther. 14, 1503-1512 (2007).

68. Akashi, H. et al. Escape from the interferon response associated with RNA interference using vectors that encode long modified hairpin-RNA. Mol. Biosyst. 1 , 382-390 (2005)

69. Konstantinova, P. et al. Inhibition of human immunodeficiency virus type 1 by RNA interference using long-hairpin RNA. Gene Ther. 13, 1403-1413 (2006).

70. Liu, Y.P., Haasnoot, J. \& Berkhout, B. Design of extended short hairpin RNAs for HIV-1 inhibition. Nucleic Acids Res. (2007).

71. Nishitsuji, H., Kohara, M., Kannagi, M. \& Masuda, T. Effective suppression of human immunodeficiency virus type 1 through a combination of short- or long-hairpin RNAs targeting essential sequences for retroviral integration. J. Virol. 80, 7658-7666 (2006)

72. Watanabe, T. et al. Intracellular-diced dsRNA has enhanced efficacy for silencing HCV RNA and overcomes variation in the viral genotype. Gene Ther. 13, 883-892 (2006).

73. Weinberg, M.S. et al. Specific inhibition of HBV replication in vitro and in vivo with expressed long hairpin RNA. Mol. Ther. 15, 534-541 (2007).

74. Boden, D., Pusch, O., Lee, F., Tucker, L. \& Ramratnam, B. Human immunodeficiency virus type 1 escape from RNA interference. J. Virol. 77, 11531-11535 (2003).

75. Wilson, J.A. \& Richardson, C.D. Hepatitis $C$ virus replicons escape RNA interference induced by a short interfering RNA directed against the NS5b coding region. J. Virol. 79, 7050-7058 (2005).

76. Wu, H.L. et al. RNA interference-mediated control of hepatitis B virus and emergence of resistant mutant. Gastroenterology 128, 708-716 (2005).

77. Gitlin, L., Stone, J.K. \& Andino, R. Poliovirus escape from RNA interference: short interfering RNA-target recognition and implications for therapeutic approaches. J. Virol. 79, 1027-1035 (2005).

78. Kusov, Y., Kanda, T., Palmenberg, A., Sgro, J.Y. \& Gauss-Muller, V. Silencing of hepatitis A virus infection by small interfering RNAs. J. Virol. 80, 5599-5610 (2006).

79. Das, A.T. et al. Human immunodeficiency virus type 1 escapes from RNA interference-mediated inhibition. J. Virol. 78, 2601-2605 (2004).

80. Arrighi, J.F. et al. DC-SIGN-mediated infectious synapse formation enhances X4 HIV-1 transmission from dendritic cells to T cells. J. Exp. Med. 200, 1279-1288 (2004)

81. Liu, S. et al. Inhibition of HIV-1 multiplication by antisense U7 snRNAs and siRNAs targeting cyclophilin A. Nucleic Acids Res. 32, 3752-3759 (2004)

82. Ping, Y.H. et al. Modulating HIV-1 replication by RNA interference directed against human transcription elongation factor SPT5. Retrovirology 1, 46 (2004).

83. Zhou, N., Fang, J., Mukhtar, M., Acheampong, E. \& Pomerantz, R.J. Inhibition of HIV-1 fusion with small interfering RNAs targeting the chemokine coreceptor CXCR4. Gene Ther. 11, 1703-1712 (2004).

84. Samson, M. et al. Resistance to HIV-1 infection in caucasian individuals bearing mutant alleles of the CCR-5 chemokine receptor gene. Nature 382, 722-725 (1996).

85. Liu, R. et al. Homozygous defect in HIV-1 coreceptor accounts for resistance of some multiply-exposed individuals to HIV-1 infection. Cell 86, 367-377 (1996).

86. Triboulet, R. et al. Suppression of microRNA-silencing pathway by HIV-1 during virus replication. Science 315, 1579-1582 (2007).

87. Morris, K.V., Chan, S.W., Jacobsen, S.E. \& Looney, D.J. Small interfering RNAinduced transcriptional gene silencing in human cells. Science 305, 1289-1292 (2004)

88. Han, J., Kim, D. \& Morris, K.V. Promoter-associated RNA is required for RNAdirected transcriptional gene silencing in human cells. Proc. Natl. Acad. Sci. USA 104, 12422-12427 (2007)

89. Weinberg, M.S. et al. The antisense strand of small interfering RNAs directs histone methylation and transcriptional gene silencing in human cells. RNA 12, 256-262 (2006).
90. Haraguchi, T. et al. SiRNAs do not induce RNA-dependent transcriptional silencing of retrovirus in human cells. FEBS Lett. 581, 4949-4954 (2007).

91. Ge, Q. et al. Inhibition of influenza virus production in virus-infected mice by RNA interference. Proc. Natl. Acad. Sci. USA 101, 8676-8681 (2004).

92. Bitko, V., Musiyenko, A., Shulyayeva, O.\& Barik, S. Inhibition of respiratory viruses by nasally administered siRNA. Nat. Med. 11, 50-55 (2005).

93. Zhang, W. et al. Inhibition of respiratory syncytial virus infection with intranasal siRNA nanoparticles targeting the viral NS1 gene. Nat. Med. 11, 56-62 (2005).

94. Rossi, J.J. RNAi as a treatment for HIV-1 infection. Biotechniques 40 (Suppl.), 25-29 (2006)

95. Westerhout, E.M., Ooms, M., Vink, M., Das, A.T. \& Berkhout, B. HIV-1 can escape from RNA interference by evolving an alternative structure in its RNA genome. Nucleic Acids Res. 33, 796-804 (2005).

96. Ter Brake, O., Konstantinova, P., Ceylan, M. \& Berkhout, B. Silencing of HIV-1 with RNA interference: a multiple shRNA approach. Mol. Ther. 14, 883-892 (2006).

97. van der Hoek, L. et al. Increased multinucleoside drug resistance and decreased replicative capacity of a human immunodeficiency virus type 1 variant with an 8-amino-acid insert in the reverse transcriptase. J. Virol. 79, 3536-3543 (2005).

98. Grimm, D. \& Kay, M.A. Combinatorial RNAi: a winning strategy for the race against evolving targets? Mol. Ther. 15, 878-888 (2007).

99. Bridge, A.J., Pebernard, S., Ducraux, A., Nicoulaz, A.L. \& Iggo, R. Induction of an interferon response by RNAi vectors in mammalian cells. Nat. Genet. 34, 263-264 (2003).

100. Jackson, A.L. \& Linsley, P.S. Noise amidst the silence: off-target effects of siRNAs? Trends Genet. 20, 521-524 (2004).

101. Grimm, D. et al. Fatality in mice due to oversaturation of cellular microRNA/short hairpin RNA pathways. Nature 441, 537-541 (2006).

102. Wang, M.B. et al. On the role of RNA silencing in the pathogenicity and evolution of viroids and viral satellites. Proc. Natl. Acad. Sci. USA 101, 3275-3280 (2004).

103. Itaya, A. et al. A structured viroid RNA serves as a substrate for dicer-like cleavage to produce biologically active small RNAs but is resistant to RNA-induced silencing complex-mediated degradation. J. Virol. 81, 2980-2994 (2007).

104. Flores, R. et al. Viroids: the minimal non-coding RNAs with autonomous replication. FEBS Lett. 567, 42-48 (2004)

105. Chang, J. \& Taylor, J.M. Susceptibility of human hepatitis delta virus RNAs to small interfering RNA action. J. Virol. 77, 9728-9731 (2003).

106. Huang, H. et al. Structure of an RNA hairpin from HRV-14. Biochemistry 40 8055-8064 (2001).

107. Rohll, J.B. et al. The $5^{\prime}$-untranslated regions of picornavirus RNAs contain inde pendent functional domains essential for RNA replication and translation. J. Virol. 68, 4384-4391 (1994).

108. Rohll, J.B., Moon, D.H., Evans, D.J. \& Almond, J.W. The $3^{\prime}$ untranslated region of picornavirus RNA: features required for efficient genome replication. J. Virol. 69 , 7835-7844 (1995).

109. Hu, W.Y., Myers, C.P., Kilzer, J.M., Pfaff, S.L. \& Bushman, F.D. Inhibition of retroviral pathogenesis by RNA interference. Curr. Biol. 12, 1301-1311 (2002).

110. Capodici, J., Kariko, K. \& Weissman, D. Inhibition of HIV-1 infection by small interfering RNA-mediated RNA interference. J. Immunol. 169, 5196-5201 (2002).

111. Surabhi, R.M. \& Gaynor, R.B. RNA interference directed against viral and cellular targets inhibits human immunodeficiency virus type 1 replication. J. Virol. 76 12963-12973 (2002)

112. Nishitsuji, H. et al. Expression of small hairpin RNA by lentivirus-based vector confers efficient and stable gene-suppression of HIV-1 on human cells including primary non-dividing cells. Microbes Infect. 6, 76-85 (2004)

113. Joshi, P.J., North, T.W. \& Prasad, V.R. Aptamers directed to HIV-1 reverse transcriptase display greater efficacy over small hairpin RNAs targeted to viral RNA in blocking HIV-1 replication. Mol. Ther. 11, 677-686 (2005).

114. Westerhout, E.M., Ter Brake, O. \& Berkhout, B. The virion-associated incoming HIV-1 RNA genome is not targeted by RNA interference. Retrovirology 3, 57-65 (2006).

115. Albertini, A.A. et al. Crystal structure of the rabies virus nucleoprotein-RNA complex Science 313, 360-363 (2006).

116. Green, T.J., Zhang, X., Wertz, G.W. \& Luo, M. Structure of the vesicular stomatitis virus nucleoprotein-RNA complex. Science 313, 357-360 (2006).

117. Fields, B.N., Raine, C.S. \& Baum, S.G. Temperature-sensitive mutants of reovirus type 3: defects in viral maturation as studied by immunofluorescence and electron microscopy. Virol. 43, 569-578 (1971).

118. Kobayashi, T., Chappell, J.D., Danthi, P. \& Dermody, T.S. Gene-specific inhibition of reovirus replication by RNA interference. J. Virol. 80, 9053-9063 (2006).

119. Geiss, B.J., Pierson, T.C. \& Diamond, M.S. Actively replicating West Nile virus is resistant to cytoplasmic delivery of siRNA. Virol. J. 2, 53 (2005).

120. Schubert, S. et al. Strand-specific silencing of a picornavirus by RNA interference: evidence for the superiority of plus-strand specific siRNAs. Antiviral Res. 73 197-205 (2007).

121. Egger, D., Teterina, N., Ehrenfeld, E. \& Bienz, K. Formation of the poliovirus replication complex requires coupled viral translation, vesicle production, and viral RNA synthesis. J. Virol. 74, 6570-6580 (2000).

122. Van Aerschot, A. Oligonucleotides as antivirals: dream or realistic perspective? Antiviral Res. 71, 307-316 (2006).

123. Neuman, B.W. et al. Inhibition, escape, and attenuated growth of severe acute respiratory syndrome coronavirus treated with antisense morpholino oligomers. J. Virol. 79, 9665-9676 (2005).

124. Matzen, K. et al. RNase $\mathrm{H}$-mediated retrovirus destruction in vivo triggered by oligodeoxynucleotides. Nat. Biotechnol. 25, 669-674 (2007). 
REVIEW

125. Ter Brake, O. \& Berkhout, B. A novel approach for inhibition of HIV-1 by RNA interterence: counteracting viral escape with a second generation of siRNAs. J. RNA Gene Silencing 1, 56-65 (2005).

126. Vitravene Study Group. Safety of intravitreous fomivirsen for treatment of cytomegalovirus retinitis in patients with AIDS. Am. J. Ophthalmol. 133, 484-498 (2002).

127. Vitravene Study Group. Randomized dose-comparison studies of intravitreous fomivirsen for treatment of cytomegalovirus retinitis that has reactivated or is persistently active despite other therapies in patients with AIDS. Am. J. Ophthalmol. 133, 475-483 (2002).

128. Vitravene Study Group. A randomized controlled clinical trial of intravitreous fomivirsen for treatment of newly diagnosed peripheral cytomegalovirus retinitis in patients with AIDS. Am. J. Ophthalmol. 133, 467-474 (2002).

129. Amado, R.G. et al. Anti-human immunodeficiency virus hematopoietic progenitor cell-delivered ribozyme in a phase I study: myeloid and lymphoid reconstitution in human immunodeficiency virus type-1-infected patients. Hum. Gene Then. 15, 251-262 (2004).

130. Macpherson, J.L. et al. Long-term survival and concomitant gene expression of ribozyme-transduced CD4+ T-lymphocytes in HIV-infected patients. J. Gene Med. 7, 552-564 (2005).

131. Levine, B.L. et al. Gene transfer in humans using a conditionally replicating lentiviral vector. Proc. Natl. Acad. Sci. USA 103, 17372-17377 (2006).

132. Range, U. et al. Enhanced T cell engraftment after retroviral delivery of an antiviral gene in HIV-infected individuals. Proc. Natl. Aced. Sci. USA 95, 1201-1206 (1998).

133. Woffendin, C., Ranga, U., Yang, Z., Xu, L. \& Mabel, G.J. Expression of a protective gene-prolongs survival of T cells in human immunodeficiency virus-infected patients. Proc. Natl. Acid. Sci. USA 93, 2889-2894 (1996).

134. Banner, I. et al. Lentiviral vector transduction of a dominant-negative rev gene into human CD 34(+) hematopoietic progenitor cells potently inhibits human immunodeficiency virus-1 replication. Mol. Sher. 15, 76-85 (2007).

135. Kohn, D.B. et al. A clinical trial of retroviral-mediated transfer of a rev-responsive element decoy gene into CD 34(+) cells from the bone marrow of human immunedeficiency virus-1-infected children. Blood 94, 368-371 (1999).

136. Anderson, J. et al. Safety and efficacy of a lentiviral vector containing three anti-HIV genes-CCR5 ribozyme, Tat-rev siRNA, and TAR Decoy-in SCID-hu mouse-derived T cells. Mol. Sher. 15, 1182-1188 (2007).

137. Morgan, R.A. et al. Preferential survival of CD4+ T lymphocytes engineered with anti-human immunodeficiency virus (HIV) genes in HIV-infected individuals. Hum. Gene Sher. 16, 1065-1074 (2005).

138. Hugle, T. \& Corny, A. Current therapy and new molecular approaches to antiviral treatment and prevention of hepatitis C. Rev. Med. Virol. 13, 361-371 (2003).

139. McHutchison, J.G. et al. A phase I trial of an antisense inhibitor of hepatitis $C$ virus (ISIS 14803), administered to chronic hepatitis C patients. J. Hepatol. 44, 88-96 (2006).

임

NATURE BIOTECHNOLOGY VOLUME 25 NUMBER 12 DECEMBER 2007

1443 